Document downloaded from:

http://hdl.handle.net/10251/100833

This paper must be cited as:

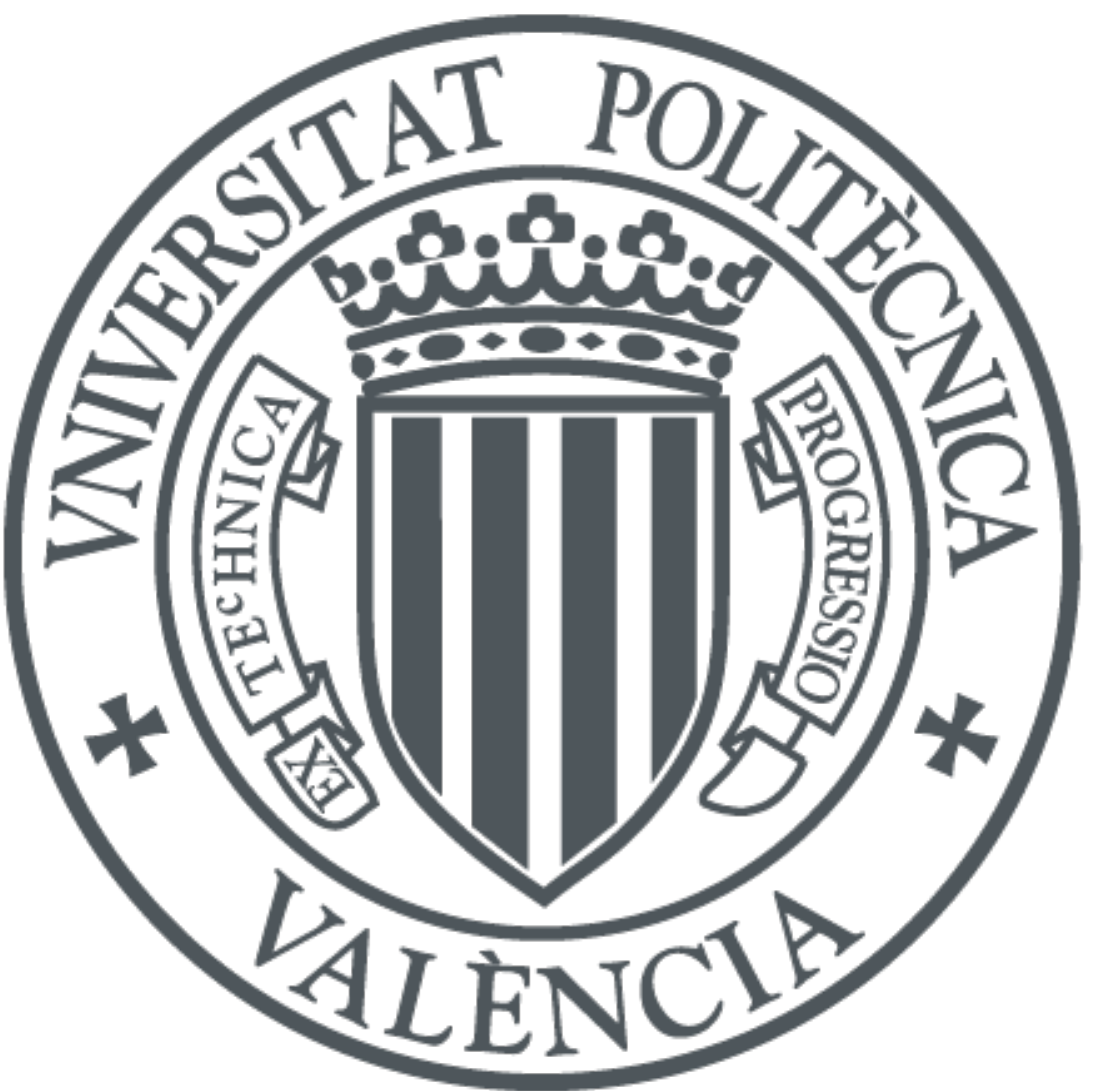

The final publication is available at

https://doi.org/10.1002/cnm.2869

Copyright John Wiley \& Sons

Additional Information 


\section{How coagulation zone size is underestimated in computer modeling of RF ablation by ignoring the cooling phase just after RF power is switched off}

Ramiro M Irastorza, ${ }^{1,2}$ Macarena Trujillo, ${ }^{3}$ Enrique Berjano. ${ }^{4}$

${ }^{1}$ Instituto de Física de Líquidos y Sistemas Biológicos (CONICET), La Plata, Argentina

${ }^{2}$ Instituto de Ingeniería y Agronomía, Universidad Nacional Arturo Jauretche, Argentina

${ }^{3}$ Instituto Universitario de Matemática Pura y Aplicada, Universitat Politècnica de València, Spain

${ }^{4}$ Biomedical Synergy, Department of Electronic Engineering, Universitat Politècnica de València, Valencia, Spain

Corresponding author: Dr. Ramiro M Irastorza, Instituto de Física de Líquidos y Sistemas Biológicos (CONICET), Calle 59 No 789, B1900BTE La Plata, Argentina. Phone: +54-2214233283, Fax:+54-221-4257317. E-mail: rirastorza@iflysib.unlp.edu.ar

Financial support: This work was supported by a grant from the "Agencia Nacional de Promoción Científica y Tecnológica de Argentina” (Ref. PICT-2012-1201), and by the Spanish "Programa Estatal de Investigación, Desarrollo e Innovación Orientada a los Retos de la Sociedad" under Grant TEC2014-52383-C3-R (TEC2014-52383-C3-1-R). 


\section{SUMMARY}

All the numerical models developed for radiofrequency (RF) ablation so far have ignored the possible effect of the cooling phase (just after RF power is switched off) on the dimensions of the coagulation zone. Our objective was thus to quantify the differences in the minor radius of the coagulation zone computed by including and ignoring the cooling phase. We built models of RF tumor ablation with two needle-like electrodes: a dry electrode (5 mm long and $17 \mathrm{G}$ in diameter) with a constant temperature protocol $\left(70^{\circ} \mathrm{C}\right)$ and a cooled electrode (30 mm long and 17G in diameter) with a protocol of impedance control. We observed that the computed coagulation zone dimensions were always underestimated when the cooling phase was ignored. The mean values of the differences computed along the electrode axis were always lower than $0.15 \mathrm{~mm}$ for the dry electrode and $1.5 \mathrm{~mm}$ for the cooled electrode, which implied a value lower than $5 \%$ of the minor radius of the coagulation zone (which was $3 \mathrm{~mm}$ for the dry electrode, and $30 \mathrm{~mm}$ for the cooled electrode). The underestimation was found to be dependent on the tissue characteristics: being more marked for higher values of specific heat and blood perfusion and less marked for higher values of thermal conductivity.

KEY WORDS: ablation, radiofrequency heating, tumor ablation 


\section{INTRODUCTION}

Radiofrequency (RF) ablation is a high-temperature focal treatment aimed at destroying irreversibly localized zones of biological tissue. Electrical current is delivered to the tissue through metal electrodes to produce a local temperature increase and the consequent creation of a thermal coagulation zone. To date, numerous theoretical models have been developed to study different issues involved in the RF ablation procedure and most of these have been based on numerical methods [1]. One of the most important functions of these models is to predict the size of the coagulation zones created. To do this, computer simulations are conducted to obtain the evolution of tissue temperature and then to estimate the thermal damage contour by using functions which take the temperature-time relationship into account [2].

To the best of our knowledge, all the computer models so far developed to predict the size of the coagulation zone created by radiofrequency energy only take the RF application phase into account when estimating the accumulated thermal damage. However, it is known that tissue temperature does not drastically decrease to a baseline, but that there is a thermal latency, which implies that temperatures at deep locations increase slightly after RF power has been switched off [3]. Furthermore, after switching off the RF power the temperature decrease is especially slow in deep tissue, where powerful heat sink mechanisms (such as the cooling inside an RF applicator during RF tumor ablation), producing a "tail" in the temperature evolution, which could contribute to the accumulated thermal damage and hence to increasing the size of the coagulation zone. Our goal was to quantify the underestimation of the coagulation zone dimensions in computer modeling studies by ignoring the cooling phase after RF power has been switched off. Although the models 
were not based on a specific tissue, the tissue properties varied within a broad range taken from literature data. We built RF ablation models for both dry and cooled needle-like electrodes and quantified the changes in the predicted coagulation zone dimensions by computer models when the cooling phase is considered in the thermal damage calculations and also identified the factors that contribute to these changes.

\section{METHODS}

\subsection{Physical situation modeled}

The physical situation modeled was a monopolar RF ablation conducted with needle-type electrodes, which are typically used in tumor ablation, assuming that the electrode was completely inserted in hepatic tissue (Figure 1A). The problem presents axial symmetry and hence a two-dimensional analysis was possible. Two types of needle-type electrodes were evaluated: dry (Fig. 1B) and internally cooled (Fig. 1C). In the former, the applied voltage was automatically modulated in order to keep the electrode temperature more or less constant. This was done with a proportional-integral (PI) control (see Section 2.4). These electrode and application modes are typically employed in bone tumor ablation [4]. In the second, an impedance control based on voltage pulses was implemented (see Section 2.5). These electrode type and application modes are typically employed in hepatic tumor ablation [5].

The dispersive electrode was modeled as an electrical condition on boundaries at a distance from the active electrode. A sensitivity analysis was used to check that tissue dimensions were large enough to keep tissue surfaces at boundary tissue temperature and voltage. The value of the maximum temperature $\left(T_{\max }\right)$ reached in the tissue at $60 \mathrm{~s}$ of $\mathrm{RF}$ 
ablation was a control parameter in these analyses. The model dimensions were considered appropriate when there was a difference of less than $0.5 \%$ in $T_{\max }$ between the simulations.

\subsection{Governing equations and model characteristics}

All the models were based on a coupled electric-thermal problem, which was solved numerically on COMSOL Multiphysics software (COMSOL, Burlington MA, USA). The governing equation for the thermal problem was the bioheat equation modified by the enthalpy method to take the vaporization phenomenon into account [6]:

$$
\frac{\partial(\rho h)}{\partial t}=\nabla \cdot(k \nabla T)+q+Q_{p}+Q_{m e t}
$$

where $\rho$ is tissue density, $h$ enthalpy, $k$ thermal conductive, $T$ temperature and $t$ time. $q$ refers to the heat source from RF power, $Q_{p}$ refers to the blood perfusion heat and $Q_{m e t}$ refers to the metabolic heat which is negligible in RF ablation. For biological tissues enthalpy is related to tissue temperature by the following expression [6]:

$$
\frac{\partial(\rho h)}{\partial t}=\frac{\partial T}{\partial t} \cdot \begin{cases}\rho_{l} c_{l} & 0<T \leq 99^{\circ} C \\ h_{f g} C & 99<T \leq 100^{\circ} C \\ \rho_{g} c_{g} & T>100^{\circ} C\end{cases}
$$

where $\rho_{i}$ and $c_{i}$ are density and specific heat of tissue respectively at temperatures below $100^{\circ} \mathrm{C}(i=l)$ and at temperatures above $100^{\circ} \mathrm{C}(i=g), h_{f g}$ is the product of water latent heat of vaporization and water density at $100^{\circ} \mathrm{C}$, and $C$ is water content inside the tissue (68\%) [7].

We simulated an in vivo situation in which $Q_{p}$ was computed from:

$$
Q_{p}=\beta \rho_{b} c_{b} \omega_{b}\left(T_{b}-T\right)
$$


where $\rho_{b}$ is density of blood, $c_{b}$ specific heat of blood, $T_{b}$ blood temperature $\left(37^{\circ} \mathrm{C}\right), \omega_{b}$ blood perfusion coefficient $\left(\omega_{b}=0.0155 \mathrm{~s}^{-1}[8]\right)$ and $\beta$ is a coefficient which took the values of 0 and 1, according to the value of the thermal damage: $\beta=0$ for $\Omega \geq 4$.6, and $\beta=1$ for $\Omega$ $<4$.6. The parameter $\Omega$ is assessed by the Arrhenius damage model [9], which associates temperature with exposure time using a first-order kinetics relationship:

$\Omega(t)=\int_{0}^{t} A e^{-\frac{\Delta E}{R T(\tau)}} d \tau$

where $R$ is the universal gas constant, $A\left(7.39 \times 10^{39} \mathrm{~s}^{-1}\right)$ is a frequency factor and $\Delta E$ $\left(2.577 \times 10^{5} \mathrm{~J} / \mathrm{mol}\right)$ is the activation energy for the irreversible damage reaction $[9,10]$. We assessed the thermal damage by the D99 isoline [11], which corresponds to $\Omega=4.6$ (99\% probability of cell death). The location of the D99 isoline defined the size of the coagulation zone. Although some previous studies have used an isotherm to compute lesion size, in the context of our work it was impossible to use the isotherm to compute the damage (as in [12]) because it does not reproduce the accumulated damage when the RF power is turned off.

The heat source from RF power $q$ (Joule losses) was given by $q=\sigma \cdot|\mathbf{E}|^{2}$, where $\mathbf{E}$ is the electric field which was obtained from the electrical problem and $\sigma$ was the electrical conductivity. The equation $\nabla \cdot \sigma \nabla V=0$ was the governing equation for the electrical problem, $V$ being the voltage. The electric field was calculated by means of $\mathbf{E}=-\nabla V$. A quasi-static approach was used since the resistive current is much lower than the displacement current at $\mathrm{RF}(\approx 500 \mathrm{kHz})$ [13]. Electrical conductivity $\sigma$ is a temperature dependent piecewise function: 


$$
\sigma(T)=\left\{\begin{array}{lc}
\sigma(37) e^{0.015(T-37)} & 0 \leq T<99^{\circ} C \\
\sigma(37) 2.5345 & 99^{\circ} C \leq T \leq 100^{\circ} C \\
\sigma(37) 2.5345-0.50183(T-100) & 100^{\circ} C<T<105^{\circ} C \\
\sigma(37) 2.5345 \times 10^{-2} & T>105^{\circ} C
\end{array}\right.
$$

where $\sigma$ (37) represents the electrical conductivity assessed at $37^{\circ} \mathrm{C}$ (see Table 1 ). Both initial and boundary temperatures were $37^{\circ} \mathrm{C}$. Tissue boundary was set at electrical insulation, excepting the bottom and top surfaces which were set at $\mathrm{V}=0$, since they represented the dispersive electrode. The delivering energy protocol depended on the electrode type (see Sections 2.4 and 2.5).

The model mesh was heterogeneous, with a finer mesh size at the electrode-tissue interface, where the highest electrical and thermal gradients were expected. All the mesh elements used were linear and triangular. An adaptive scheme was used for the time step, since we let the time-stepping method chose time steps freely. The size of the finer mesh and the optimal time-step was estimated by a sensitivity analysis on the same lines as that used for the outer geometry dimensions.

\subsection{Tissue characteristics}

The characteristics of materials used in the model are shown in Table 1, including the metallic electrode and the plastic partially covering the electrode [7,14]. We also assessed the effect of changing the tissue characteristics expected to affect the temperature distributions (and hence the size of the coagulation zone) during tPOST-RF: thermal conductivity, specific heat, and blood perfusion. The values were varied in the range reported in the literature [14]. Specifically, we selected three values for each parameter: 
maximum, minimum, and intermediate. The minimum values for thermal conductivity and specific heat were those of low water content tissue (such as fat, bone marrow, and cortical bone), while the maximum values for thermal conductivity and specific heat were those of high water content tissue (e.g. vitreus humor). We also considered an intermediate value which was that of liver. Since it is known that that the blood perfusion value can be markedly affected by the target location, affections, and tumors, we varied the value according to literature data in the case of liver tissue $[15,16]$. Twenty-seven simulations were conducted with all the combinations of minimum, intermediate, and maximum values of thermal conductivity $k(0.2,0.52$, and $0.6 \mathrm{~W} / \mathrm{m} \cdot \mathrm{K}$, respectively), specific heat $c(1313$, 3540, and $4000 \mathrm{~J} / \mathrm{kg} \cdot \mathrm{K}$, respectively), and blood perfusion $\omega_{b}\left(0.009,0.0155\right.$, and $0.0285 \mathrm{~s}^{-}$ ${ }^{1}$, respectively).

\subsection{Assessment of the impact of post-RF time}

The impact of considering the cooling phase after RF power is switched-off ( $t_{\text {POST-RF }}$ ) was studied by comparing the coagulation zone dimensions at this point in time $\left(\mathrm{t}_{\mathrm{ON}-\mathrm{RF}}\right)$ with those taken after a longer duration ( $\left.\mathrm{t}_{\mathrm{ON}-\mathrm{RF}}+\mathrm{t}_{\mathrm{POST}-\mathrm{RF}}\right)$, which was the time until tissue temperature returned to baseline $\left(\approx 37^{\circ} \mathrm{C}\right)$, i.e. the upper bound limit $t$ in the integral of the Eq. (4) is really that of either the active ablation time ( $\left.t_{\mathrm{ON}-\mathrm{RF}}\right)$ or total time $\left(\mathrm{t}_{\mathrm{ON}-\mathrm{RF}}+\mathrm{t}_{\mathrm{POST}-}\right.$ $\mathrm{RF})$. All the simulations showed that at $400 \mathrm{~s}$ after RF application the difference between the highest temperature and baseline was around $0.004^{\circ} \mathrm{C}$, so that we considered $t_{\mathrm{POST}-\mathrm{RF}}=800$ s as reasonable. Coagulation zone dimensions were compared by the Arrhenius model. The main idea was to calculate the difference between the electrode-D99 distances computed at $\mathrm{t}_{\mathrm{ON}-\mathrm{RF}}$ and $\mathrm{t}_{\mathrm{ON}-\mathrm{RF}}+\mathrm{t}_{\mathrm{POST}-\mathrm{RF}}$. The measurement was taken from the region of the cylindrical 
part of the active electrode, without considering contact with the insulated part or the distal tip of the active electrode (see Fig. 2a). The difference in the damage was computed by subtracting the coordinates $r$ of the contours ( $\left.r_{\text {post }}-r_{\text {on }}\right)$ along the $z$ axis (Fig. 2c). Both $r_{\text {post }}$ and $r_{\text {on }}$ are really transverse diameters of the coagulation zone created with a needle-like electrode. They are also known as minor diameters, as opposed to the major diameters, which are parallel to the electrode axis. The mean value of the difference between $r_{\text {post }}$ and $r_{\text {on }}$ was computed. The graph in Fig. 2c represents the isolines shown in Fig. 2b.

\subsection{Needle-type dry electrode and constant temperature protocol}

We first modeled a non-cooled (dry) electrode working in a constant temperature mode with a target temperature of $70^{\circ} \mathrm{C}$ using a PI controller similar to the one described in [17]. Briefly, a dynamical system was considered in which the input variable was the voltage applied to the electrode, and the output variable was the temperature measured at the tip of the electrode. There are different ways of controlling tip temperature, the most common strategy being proportional-integral-derivative (PID) control. Here, we tuned a PI controller that reached the target temperature within 20 seconds without overshooting. Using intermediate values of the physical properties of the tissue, we obtained $\mathrm{K}_{\mathrm{p}}=1.15^{\circ} \mathrm{C} / \mathrm{V}$ y $\mathrm{K}_{\mathrm{i}}$ $=0.1^{\circ} \mathrm{C} / \mathrm{V} \cdot \mathrm{s}$. Ablation duration ( $\mathrm{t}_{\mathrm{ON}-\mathrm{RF}}$ ) was $240 \mathrm{~s}$, electrode length $5 \mathrm{~mm}$ and electrode

diameter 17G (1.473 mm), parameters typically employed in RF ablation of musculoskeletal tumors.

\subsection{Cooled needle-type electrode and impedance control protocol}


Long internally cooled electrodes are used for RF ablation of tumors in soft organs such as the liver. The most typical of these electrodes is the Cool-Tip device (Covidien, Boulder, CO, USA), with a $17 \mathrm{G}$ electrode $(1.473 \mathrm{~mm}$ diameter) and $3 \mathrm{~cm}$ in length. The cooling effect of the liquid circulating inside the electrode was modeled using a thermal convection coefficient $h_{r}$ with a value of $3,127 \mathrm{~W} / \mathrm{K} \cdot \mathrm{m}^{2}$ and a coolant temperature of $5^{\circ} \mathrm{C}$, following Newton's Law of Cooling. The value of $h_{r}$ was calculated by considering the electrode length $(3 \mathrm{~cm})$ and a flow rate of $45 \mathrm{~mL} / \mathrm{min}$ through an area equivalent to half the cross section of the inner diameter of the electrode.

An impedance-controlled pulsing protocol was modeled for a $240 \mathrm{~s}$ ablation with a current constant of $1,500 \mathrm{~mA}$, which is aimed at increasing coagulation zone size, since periods of low current deposition alternate with higher peak current, allowing the tissue near the electrode to cool while the deeper tissue is heated [18]. RF power was switched off once the impedance exceeded a threshold, or roll-off, which is usually $20 \Omega$ higher than the initial value, and was switched on again after 15 s [19].

\section{RESULTS}

Figure 3 shows the mean value of the difference in damage computed along the $z$ axis for the needle-type dry electrode and constant temperature protocol when the tissue characteristics were changed. Overall, the most important finding was that the differences between considering and ignoring the cooling phase in the computer simulations were always lower than $0.15 \mathrm{~mm}$ (in terms of coagulation zone radius), which represented approximately $5 \%$ of the radius of the coagulation zone (around $3 \mathrm{~mm}$ ). However, in most cases the difference was even lower than $0.1 \mathrm{~mm}(\approx 3.3 \%)$. 
In the simulations of a needle-type cooled electrode with an impedance control protocol, there were around 6 roll offs throughout the 240 s of ablation. Figure 4 shows the mean value of the difference in damage computed along the $z$ axis for the needle-type cooled electrode with an impedance control protocol when tissue characteristics were varied. In this case, the differences between considering and ignoring the cooling phase in the computer simulations were always lower than $1.5 \mathrm{~mm}$ (in terms of radius of coagulation zone), which represented around 5\% of the radius of the coagulation zone (around $30 \mathrm{~mm}$ ). However, in most cases the difference was less than $1 \mathrm{~mm}(\approx 3.3 \%)$.

Regarding the effect of changing the tissue characteristics, the findings were qualitatively similar for both electrode designs and protocols. Higher values of thermal conductivity involved slightly lower $r_{\text {post }}-r_{\text {on }}$ differences (see the trend lines in Figs. 3a and 4a). However, the changes in blood perfusion and specific heat showed a more marked effect. The $r_{\text {post }}-r_{\text {on }}$ differences were considerably lower for higher values of blood perfusion, and increased for higher values of specific heat.

Finally, the effect of ablation time was also assessed by analyzing the $r_{\text {post }}-r_{\text {on }}$ difference throughout the ablation period (each $60 \mathrm{~s}$ ). We did not find any important differences or any perceptible trend over time.

\section{DISCUSSION}

\subsection{Review of the results}

As the numerical modeling technique applied to ablative therapies based on hightemperature is intended to accurately predict thermal coagulation zones, the models should hence be built on a solid foundation of realism. One of the issues that have been 
traditionally ignored in numerical modeling is the role played by the remaining heat stored in the tissue once RF power is switched off. All the computer modeling studies to date have considered only a simulation time corresponding with the duration of the RF power application, and therefore quantified the coagulation zone dimensions at the end of this period, which clearly ignores the possible effect of the remaining heat to slightly increase the final size of the coagulation zone.

Our goal was to compare the coagulation zone dimensions, especially the minor radius, created by considering and ignoring this remaining heat. We focused on the destruction of tumors, which is one of the most important applications in RF ablation. In this context, we considered two electrode designs (dry and cooled), each one with their particular protocol to deliver RF power (constant temperature and impedance control, respectively). In both cases we observed that the impact of ignoring the cooling phase in the computer simulations meant that the minor radius of the coagulation zone was underestimated by less than $6.6 \%$ and $12 \%$ for dry and cooled electrodes, respectively. The clinical impact of this finding depends on the specific objective of each modeling study. For instance, it could be considered to be negligible in the case of comparative studies in which the absolute values of coagulation zone dimensions are not important. In contrast, a difference of $5 \%$ could be important when the numerical model is employed to accurately predict the coagulation zone dimensions.

This underestimation was dependent on the tissue characteristics in a way that can be explained in physical terms. The cases in which the underestimation in the coagulation zone dimension is higher corresponded with those in which the remaining heat is evacuated more slowly. This occurs when: 1) the tissue has more capacity to store heat, which is 
equivalent to having a high value of specific heat and/or of density (issue not evaluated in this study, since all biological tissues have a similar density, around 1,000 kg/m ${ }^{3}$ ); 2) heat cannot be easily conducted through the tissue, which is equivalent to having a low value of thermal conductivity; and 3) the heat evacuation mechanisms are poor, which is equivalent to having a low blood perfusion value.

\subsection{Limitations of the study}

This study has certain limitations which need to be emphasized: firstly, the differences in coagulation zone dimensions between considering and ignoring the cooling phase were exclusively evaluated in terms of the thermal coagulative necrosis created by the direct action of heat, i.e. using the Arrhenius damage model, which associates temperature with exposure time (Eq. 4). In a real situation, the coagulation zone finally created would include a thin rim comprised of cells which would die by apoptosis (maybe caused by thermal shock) rather than by thermal coagulative necrosis. This issue is outside the scope of this study, which only focused on the role of the remaining heat in cell death.

Secondly, we only considered the case of RF tumor ablation with a needle-like electrode, although other settings and electrode designs are also possible in RF ablation numerical modeling. For instance, in RF ablation of cardiac arrhythmias, a semispherical electrode embedded at the tip of an intravascular catheter is placed on the endocardium. In this case, dry and cooled electrodes can also be employed, along with different protocols for delivering RF power (constant temperature and constant power). Even though RF cardiac ablation was not considered in this study, we do not think that values higher than $5 \%$ can be observed in the underestimation of the coagulation zone dimensions. In our 
opinion, remaining heat could be rapidly evacuated towards the circulating blood through the electrode body and through the endocardium-blood interface, which would reduce the difference between $r_{\text {post }}$ and $r_{\text {on }}$.

\subsection{Clinical impact of the findings}

Apart from a scientific interest in the effect of the cooling phase on the computed damage produced by thermal therapy, it is also of interest to consider the real clinical impact of an estimation error of $5 \%$ (equivalent to $3 \mathrm{~mm}$ in the case of a 3-mm long RF electrode). This is important in the context of computer-aided planning and simulation tools and imageguided ablative procedures. In this respect, although the current electromagnetic navigation systems offer tracking errors of up to $5 \mathrm{~mm}$ [20], in the near future imaging systems are expected to offer an improved resolution of even less than 300 microns [21]. For instance, in the context of RF ablation of the atrial wall, the mathematical processing of a finite element model based on images from computed tomographic angiography has already made it possible to estimate the atrial wall thickness to within less than $0.2 \mathrm{~mm}$ [22]. It is precisely in the field of RF heating of small volume targets (such as the atrial wall) where an underestimation error of $5 \%$ could have serious consequences. In fact, it is in this field, in which optical techniques offer extremely high spatial resolution, that they are being proposed to map thermal lesions [23].

\section{CONCLUSIONS}

Ignoring the cooling phase that occurs just after RF power has been switched off always involves underestimating the computed coagulation zone dimensions. This underestimation 
was always lower than $5 \%$ of the coagulation zone radius for both dry and cooled electrodes and was dependent on tissue characteristics: it was more marked for higher values of specific heat and blood perfusion, and less marked for higher values of thermal conductivity.

\section{REFERENCES}

1. Berjano EJ. Theoretical modeling for radiofrequency ablation: state-of-the-art and challenges for the future. Biomed Eng Online. 2006 Apr 18;5:24. doi: 10.1186/1475-925X-5-24.

2. Pearce JA. Comparative analysis of mathematical models of cell death and thermal damage processes. Int J Hyperthermia. 2013 Jun;29(4):262-80. doi: 10.3109/02656736.2013.786140.

3. Wittkampf FH, Nakagawa H, Yamanashi WS, Imai S, Jackman WM. Thermal latency in $\begin{array}{lllll}\text { radiofrequency } & \text { ablation. } & \text { Circulation. } & 1996 \quad \text { 15;93(6):1083-6. }\end{array}$ http://circ.ahajournals.org/content/93/6/1083.long, Accessed August 27, 2016.

4. Pinto W R, Taminiau C H, Vanderschueren A H M, Hogendoorn G M, Bloem P C W and Obermann J L Perspective. Technical considerations in CT-guided radiofrequency thermal ablation of osteoid osteoma: Tricks of the trade. American Journal of Roentgenology, 2002, 179, 1633-42. doi: 10.2214/ajr.179.6.1791633.

5. Fukushima T, Ikeda K, Kawamura Y, et al. Randomized controlled trial comparing the efficacy of impedance control and temperature control of radiofrequency interstitial thermal ablation for treating small hepatocellular carcinoma. Oncology 2015,89(1):47-52. doi: 10.1159/000375166.

6. Abraham JP, Sparrow EM. A thermal-ablation bioheat model including liquid-to-vapor phase change, pressure- and necrosis-dependent perfusion, and moisture-dependent properties. Int J Heat Mass Transfer 2007;50:2537-44. doi: 10.1016/j.ijheatmasstransfer.2006.11.045.

7. Pätz T, Kröger T, Preusser T. Simulation of radiofrequency ablation including water evaporation. IFMBE Proceedings 2009;25/IV:1287-90. 
8. Hall SK, Ooi EH, Payne SJ. Cell death, perfusion and electrical parameters are critical in models of hepatic radiofrequency ablation. Int $J$ Hyperthermia. 2015;31(5):538-50. doi: 10.3109/02656736.2015.1032370.

9. Chang IA. Considerations for thermal injury analysis for RF ablation devices. Biomed Eng Online 2010;4:3-12. doi: 10.2174/1874120701004020003.

10. Kim BM, Jacques S, Rastegar S, Thomsen S, Motamedi M. Nonlinear finite-element analysis of the role of dynamic changes in blood perfusion and optical properties in laser coagulation of tissue. IEEE J Sel Top Quantum Electron. 1996;2:922-33. http://ieeexplore.ieee.org/document/577317/

11. Chang IA and Nguyen UD. Thermal modeling of lesion growth with radiofrequency ablation devices. BioMedical Engineering OnLine. 2004, Aug 3:27. doi: http://dx.doi.org/10.1186/1475-925X-3-27.

12. Schutt DJ and Haemmerich D, Effects of variation in perfusion rates and of perfusion models in computational models of radio frequency tumor ablation, Medical physics 2008 Jul 35 3462-3470. doi: http://dx.doi.org/ 10.1118/1.2948388.

13. Doss JD. Calculation of electric fields in conductive media. Med Phys 1982;9:566-73. doi: $10.1118 / 1.595107$

14. Hasgall PA, Di Gennaro F, Baumgartner C, et al. "IT’IS Database for thermal and electromagnetic parameters of biological tissues,” Version 3.0, September 01st, 2015, doi: 10.13099/VIP21000-03-0. www.itis.ethz.ch/database. Accessed August 27, 2016.

15. Pandharipande PV, Krinsky GA, Rusinek H, \& Lee VS. Perfusion imaging of the liver: current challenges and future goals. Radiology. 2005 Mar 23, 661-673. doi: http://dx.doi.org/10.1148/radiol.2343031362.

16. Tsushima Y, Funabasama S, Aoki J, Sanada S, \& Endo K. Quantitative perfusion map of malignant liver tumors, created from dynamic computed tomography data. Academic radiology. 2004 Sep 15, 215-223. doi: http://dx.doi.org/10.1016/S1076-6332(03)00578-6.

17. Irastorza RM, Trujillo M, Martel Villagrán J, Berjano E. Computer modelling of RF ablation in cortical osteoid osteoma: Assessment of the insulating effect of the reactive zone. Int J Hyperthermia. 2016 May;32(3):221-30. doi: http://dx.doi.org/10.3109/02656736.2015.1135998. 
18. Goldberg SN, Stein MC, Gazelle GS, Sheiman RG, Kruskal JB, Clouse ME. Percutaneous radiofrequency tissue ablation: optimization of pulsed-radiofrequency technique to increase coagulation necrosis. J Vasc Interv Radiol. 1999 Jul-Aug;10(7):907-16. doi: http://dx.doi.org/10.1016/S1051-0443(99)70136-3.

19. Trujillo M, Bon J, José Rivera M, Burdío F, Berjano E. Computer modelling of an impedancecontrolled pulsing protocol for RF tumour ablation with a cooled electrode. Int J Hyperthermia. 2016 Jul 24:1-9. doi: http://dx.doi.org/10.1080/02656736.2016.1190868.

20. Venkatesan AM, Kadoury S, Abi-Jaoudeh N, Levy EB, Maass-Moreno R, Krücker J, Wood BJ. Realtime FDG PET guidance during biopsies and radiofrequency ablation using multimodality fusion with electromagnetic navigation. $\quad$ Radiology. $2011 \quad$ May $\quad 4: \quad 848-856 . \quad$ doi: http://dx.doi.org/10.1148/radiol.11101985.

21. Ertürk MA, Hegde SS, Bottomley PA. Radiofrequency Ablation, MR Thermometry, and High-SpatialResolution MR Parametric Imaging with a Single, Minimally Invasive Device. Radiology. 2016 Mar 30. doi: http://dx.doi.org/10.1148/radiol.2016151447.

22. Bishop M, Rajani R, Plank G, Gaddum N, Carr-White G, Wright M, Niederer S. Three-dimensional atrial wall thickness maps to inform catheter ablation procedures for atrial fibrillation. Europace. 2016 Apr 4: 376-383. doi: http://dx.doi.org/10.1093/europace/euv073.

23. Wang H, Kang W, Carrigan T, Bishop A, Rosenthal N, Arruda M, Rollins AM. In vivo intracardiac optical coherence tomography imaging through percutaneous access: toward image-guided radiofrequency ablation. Journal of biomedical optics. 2011 Nov 8: 110505-1-3. doi: http://dx.doi.org/10.1117/1.3656966. 
Table 1. Characteristics of the materials used in the theoretical model $[7,8]$.

\begin{tabular}{|l|l|l|l|l|}
\hline \multicolumn{1}{|c|}{ Material } & $\boldsymbol{\rho}\left(\mathbf{k g} / \mathbf{m}^{3}\right)$ & \multicolumn{1}{|c|}{$\boldsymbol{c ( \mathbf { J } / \mathbf { k g } \cdot \mathbf { K } )}$} & $\boldsymbol{k}(\mathbf{W} / \mathbf{m} \cdot \mathbf{K})$ & $\boldsymbol{\sigma}(\mathbf{S} / \mathbf{m})$ \\
\hline Tissue & $1079^{(1)}$ & $3540^{(1)}[1313-4000]^{(3)}$ & $0.52[0.2-0.6]^{(3)}$ & $0.148^{(4)}$ \\
\cline { 2 - 4 } & $370^{(2)}$ & $2156^{(2)}$ & & \\
\hline Electrode & $8 \times 10^{3}$ & 480 & 15 & $7.4 \times 10^{6}$ \\
\hline Plastic & 70 & 1045 & 0.026 & $1 \times 10^{-5}$ \\
\hline Blood & 1000 & 3639 & & \\
\hline
\end{tabular}

$\rho$, density; $c$, specific heat; $k$, thermal conductivity; $\sigma$, electric conductivity;

${ }^{(1)} \mathrm{At}$ temperature below $100^{\circ} \mathrm{C} .{ }^{(2)} \mathrm{At}$ temperature above $100^{\circ} \mathrm{C}$.

${ }^{(3)}$ Minimum and maximum values for the sensitivity analysis (see section 2.3 for more details).

${ }^{(4)}$ Assessed at $37^{\circ} \mathrm{C}$. 


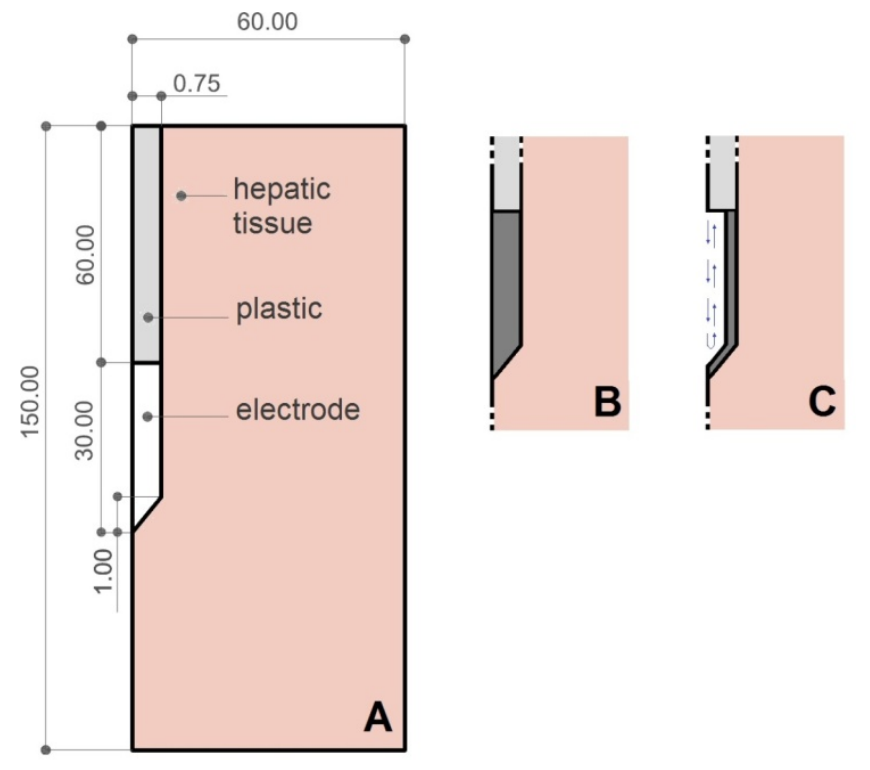

Figure 1 A: Geometry of the model used in the first set of simulations (out of scale, dimensions in mm). The outer dimensions (60 mm wide and $150 \mathrm{~mm}$ long) were calculated by means of a sensitivity analysis. B: Detail of dry electrode geometry. C: Detail of internally cooled electrode geometry. 
(a)
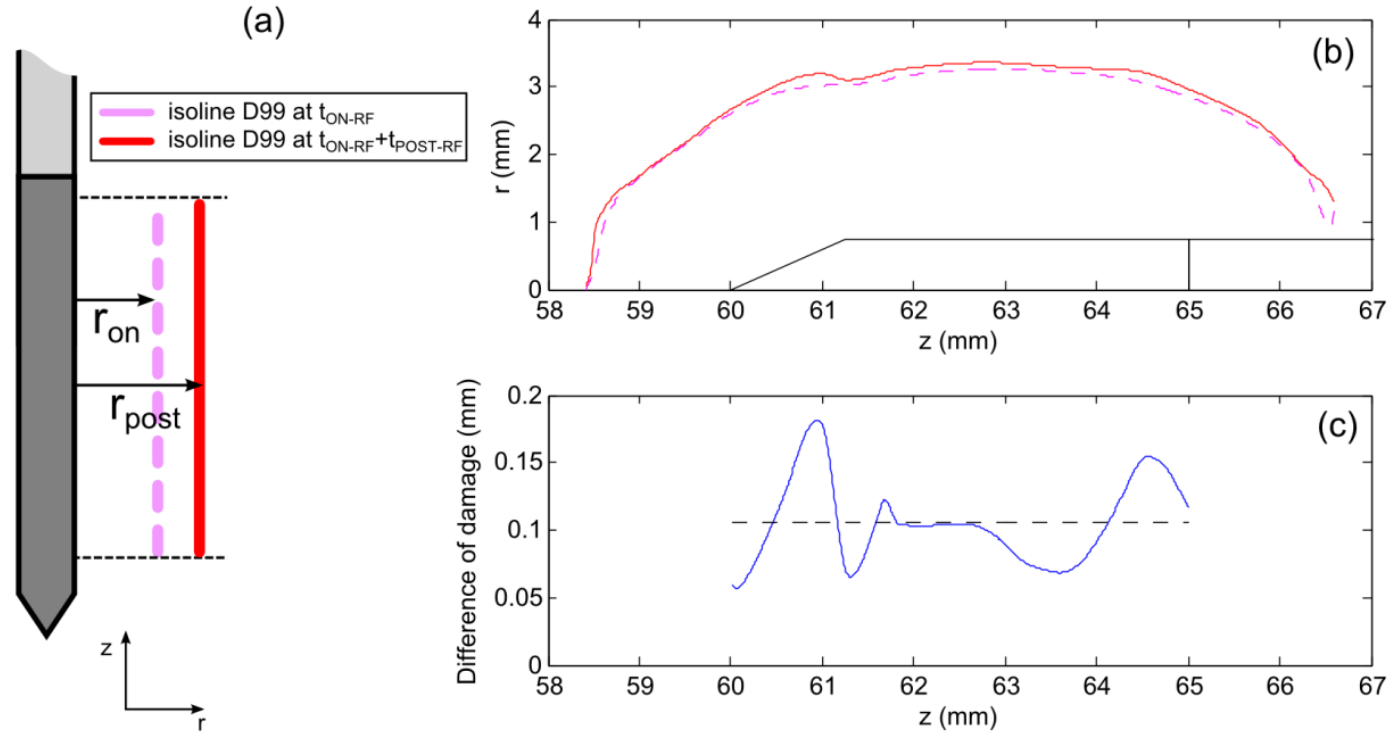

Figure 2 Procedure to compare the coagulation zones created with and without considering $\mathrm{t}_{\mathrm{POST} \text {-RF }}$ in the simulations. (a) Scheme of the variables under study. (b) Isolines D99 at ton-RF (dashed line) and at $\mathrm{t}_{\mathrm{ON}-\mathrm{RF}}+\mathrm{t}_{\mathrm{POST}-\mathrm{RF}}$ (continuous line). (c) Difference of damage $\left(r_{\mathrm{post}}-r_{\mathrm{on}}\right)$ along the electrode (coordinate $z$ ). The dashed line shows the mean value, which is considered in the subsequent analyses. 

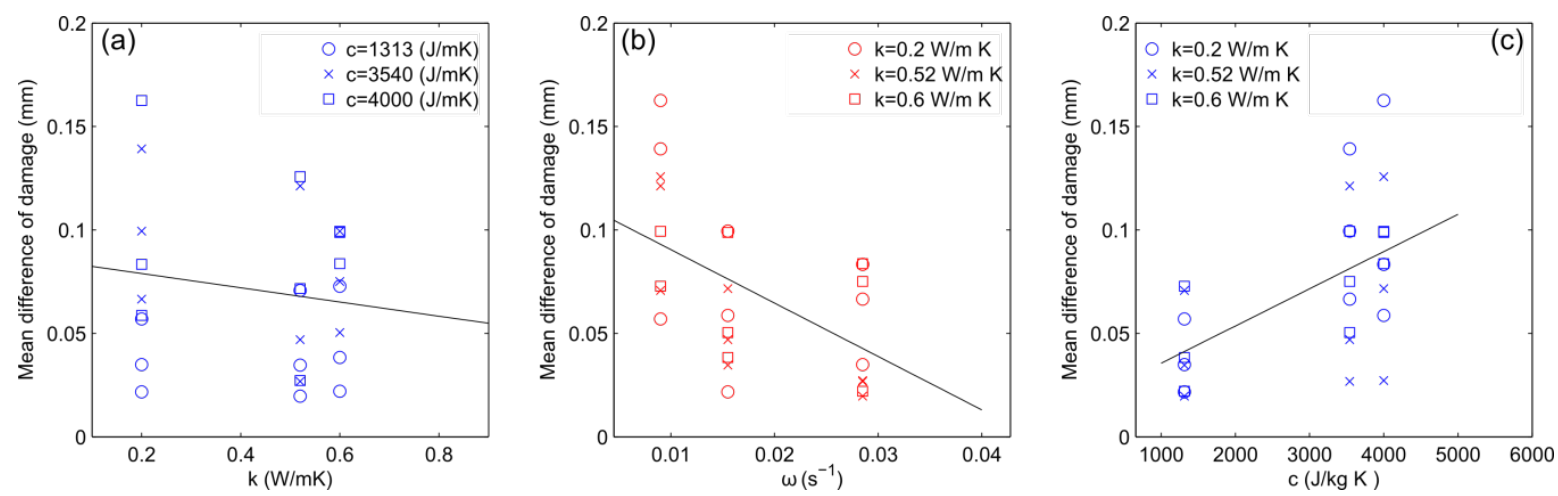

Figure 3 Mean values of the difference of thermal damage $\left(r_{\text {post }}-r_{\text {on }}\right)$ along the electrode axis (coordinate $z$ ) between considering $\left(r_{\text {post }}\right)$ and ignoring $\left(r_{\text {on }}\right)$ the cooling phase in the simulations. The results correspond to the case of needle-type dry electrode and constant temperature protocol, and for different values of the tissue characteristics: thermal conductivity $(k)$, blood perfusion $(\omega)$ and specific heat $(c)$. The value shown in Table 1 was considered if not otherwise indicated. 

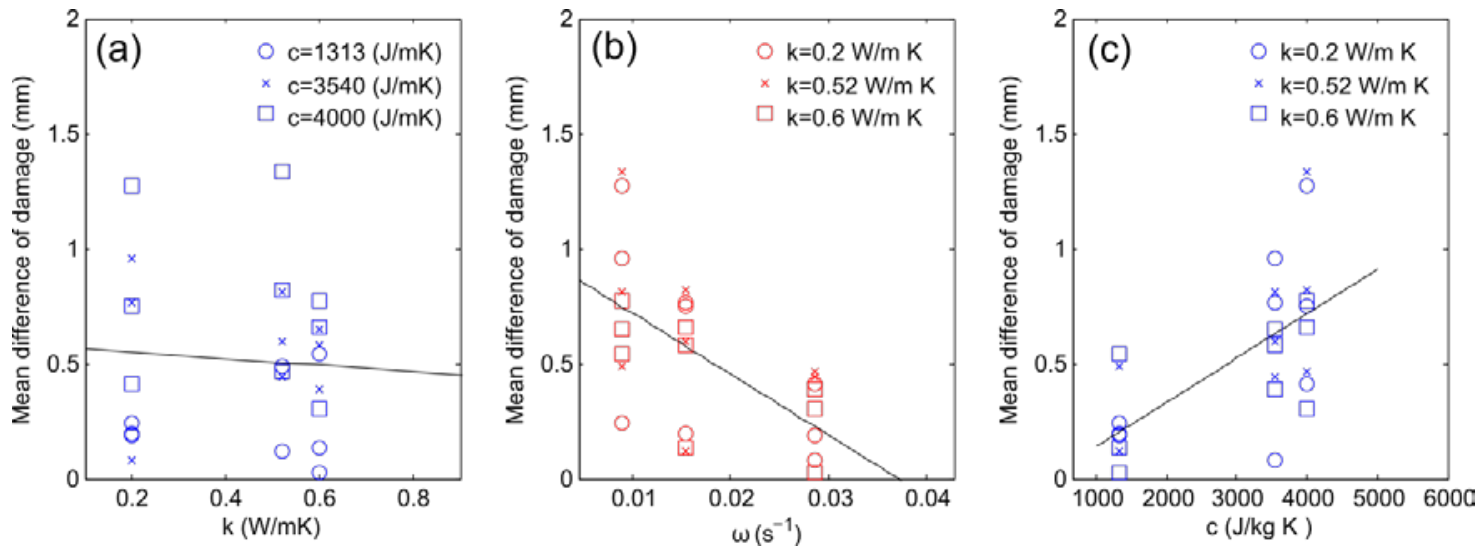

Figure 4 Mean values of the difference of thermal damage $\left(r_{\text {post }}-r_{\text {on }}\right)$ along the electrode axis (coordinate $z$ ) between considering $\left(r_{\text {post }}\right)$ and ignoring $\left(r_{o n}\right)$ the cooling phase in the simulations. The results correspond to the case of needle-type cooled electrode and impedance control protocol, and for different values of the tissue characteristics: thermal conductivity $(k)$, blood perfusion $(\omega)$ and specific heat $(c)$. The value shown in Table 1 was considered if not otherwise indicated. 